\title{
Mutational spectrum by phenotype: panel-based NGS testing of patients with clinical suspicion of RASopathy and children with multiple café-au-lait macules
}

\section{Mutation spectrum in NF1-related phenotypes}

Elisabeth Castellanos ${ }^{1 *}$, Inma Rosas ${ }^{1}$, Alejandro Negro ${ }^{1}$, Bernat Gel ${ }^{1}$, Andreu Alibés ${ }^{2}$, Neus Baena ${ }^{3}$, Mercè Pineda

4, Graciela $\mathrm{Pi}^{5}$, Guillem Pintos ${ }^{6}$, Hector Salvador ${ }^{7}$, Conxi Lazaro ${ }^{8,12}$, Ignacio Blanco ${ }^{9}$, Lluïsa Vilageliu ${ }^{10}$, Hilde Brems $^{11}$, Daniel Grinberg ${ }^{10}$, Eric Legius ${ }^{11}$, Eduard Serra ${ }^{1,12^{*}}$

1. Hereditary Cancer Group, Program of Predictive and Personalized Medicine of Cancer (PMPPC), Germans Trias \& Pujol Research Institute (IGTP), Badalona, Barcelona, Spain

2. Program of Predictive and Personalized Medicine of Cancer (PMPPC), Germans Trias \& Pujol Research Institute (IGTP), Badalona, Barcelona, Spain

3. Genetics Laboratory of the UDIAT-CD, Parc Tauli Health Corporation, Sabadell, Barcelona, Spain

4. Neuropaediatrics Unit, Hospital Sant Joan de Déu, Esplugues, Barcelona, Spain

5. Neuropaediatrics Unit, La Ribera Hospital, Valencia, Spain

6. Department of Pediatrics, Germans Trias i Pujol University Hospital and Research Institute (IGTP), Badalona, Universitat de Barcelona, Spain

7. Paediatrics Oncology Unit, Hospital Sant Joan de Déu, Esplugues, Barcelona, Spain

8. Hereditary Cancer Program, Catalan Institute of Oncology (ICO-IDIBELL-ONCOBELL), CIBERONC, L'Hospitalet de Llobregat, Barcelona, Spain

9. Clinical Genetics and Genetic Counseling Program, Germans Trias i Pujol Hospital, Can Ruti Campus, Badalona, Barcelona, Spain

10. Department of Genetics, Microbiology and Statistics, Facultat de Biologia, Universitat de Barcelona (UB), IBUB, IRSJD, CIBERER, Barcelona, Spain

11. Laboratory for Neurofibromatosis Research, Department of Human Genetics, KU Leuven University Hospital, Belgium

12. Centro de Investigación Biomédica en RED (CIBERONC), Instituto de Salud Carlos III, Madrid, Spain.

\section{ACKNOWLEDGEMENTS:}

We are indebted to the IGTP Translational Genomics Core Facility and staff for their technical support. We thank Dr. Gabau and Dra. Guitart from UDIAT-CD, Parc Taulí Health Corporation, for their contribution with clinical cases. We would like to acknowledge the constant support of the different NF lay associations: Asociación de Afectados de Neurofibromatosis and ACNefi.

This work has been supported by: the Spanish Ministry of Science and Innovation, Carlos III Health Institute (ISCIII) (PI11/1609; PI14/00577) (RTICC RD12/0036/008), CIBERONC, Plan Estatal de I + D + I 2013-2016, and co-financed by the FEDER program; and the Government of Catalonia (2014 SGR 338; 2017 SGR 00496), CERCA Program.

The data that support the findings of this study are available on request from the corresponding author. Some data are not publicly available due to privacy or ethical restrictions. The authors declare no conflicts of interests.

This article has been accepted for publication and undergone full peer review but has not been through the copyediting, typesetting, pagination and proofreading process which may lead to differences between this version and the Version of Record. Please cite this article as doi: $10.1111 /$ cge.13649 


\begin{abstract}
Children with Neurofibromatosis Type 1 (NF1) may exhibit an incomplete clinical presentation, making difficult to reach a clinical diagnosis. A phenotypic overlap may exist with children with other RASopathies or with other genetic conditions if only multiple café-au-lait macules (CALMs) are present. The syndromes that can converge in these inconclusive phenotypes have different clinical courses. In this context, an early genetic testing has been proposed to be clinically useful to manage these patients. We present the validation and implementation into diagnostics of a custom NGS panel (I2HCP) for testing patients with a clinical suspicion of a RASopathy (n=48) and children presenting multiple CALMs $(\mathrm{n}=102)$. We describe the mutational spectrum and the detection rates identified in these two groups of individuals. We identified pathogenic variants in 21 out of 48 patients with clinical suspicion of RASopathy, with mutations in NF1 accounting for $10 \%$ of cases. Furthermore, we identified pathogenic mutations mainly in the NF1 gene, but also in SPRED1, in more than $50 \%$ of children with multiple CALMs, exhibiting a NF1 mutational spectrum different from a group of clinically diagnosed NF1 patients ( $\mathrm{n}=80)$. An NGS panel strategy for the genetic testing of these two phenotype-defined groups outperforms previous strategies.
\end{abstract}

KEY WORDS: Neurofibromatosis type 1, RASopathies, genetic testing, NGS panel, multiple CALMs 


\section{INTRODUCTION}

Neurofibromatosis Type 1 (NF1) is a neuro-cutaneous genetic disease with a birth incidence of 1:2500-1:3000 ${ }^{1}$. It is characterized by a highly variable expressivity, in which multiple pigmented café-au-lait macules (CALMs) in the skin ( $>0,5 \mathrm{~cm}$ in children; $>1,5 \mathrm{~cm}$ in adults) and multiple cutaneous neurofibromas affect almost all NF1 individuals. Clinical criteria are normally fulfilled at the age of $8^{2}$ although cutaneous neurofibromas are not developed until late teens ${ }^{3}$ turning out in inconclusive clinical diagnostic of NF1 in childhood.

The presence of few skin CALMs at an early age is common among children ${ }^{4,5}$ but in multiple form could also be an indication of the presence of an inherited disease. The solely presence of 6 or more CALMs at early childhood constitutes a high risk for having NF1 and it is one of the main clinical criteria used ${ }^{6-8}$. However, multiple CALMs could also be indicative of other monogenic diseases, like some RASopathies or other syndromes like PTEN hamartoma tumor or Cowden syndrome (PHTS), Carney Syndrome or constitutive mismatch repair deficiency (CMMRD) $)^{4,5,9,10}$.

The RASopathies constitute a clinically defined group of genetic conditions caused by germline mutations in genes that encode for components of the RAS/mitogen-activated protein kinase (MAPK) pathway. RASopathies include in addition to NF1: Noonan syndrome (NS), Noonan syndrome with multiple lentigines (NSML), Costello syndrome (CS), Legius Syndrome (LS), cardio-facio-cutaneos (CFC) syndrome and capillary malformation- arteriovenous malformation (CM-AVM) ${ }^{11}$. Each RASopathy exhibits a particular group of clinical manifestations, but due to the common underlying RAS/MAPK pathway deregulation, many of these conditions exhibit numerous overlapping phenotypic features, especially during early childhood (reviewed in ${ }^{12}$ ). Overlapping clinical manifestations include cutaneous, musculoskeletal, and ocular abnormalities; craniofacial dysmorphology; cardiovascular abnormalities; neurocognitive impairment; hypotonia; and increased risk of tumor development. NF1, Legius syndrome and CMAVM are caused by loss of function mutations in $N F 1^{13}$, SPRED $1^{14}$ or $R A S A 1$ genes ${ }^{15}$, respectively. Others like Noonan, NSML and CFC syndromes exhibit genetic heterogeneity and together with Costello syndrome are caused by activating mutations in PTPN11, SOS1, RAF1, BRAF, KRAS, NRAS, SHOC2, BRAF, MAP2K1, MAP2K2, CBL,

HRAS and RIT1 $1^{11,16-19}$. There are evidences that support other genes to be potentially associated with RASopathies (reviewed in ${ }^{17}$ ) like $R A S A 2^{20}, A 2 M L 1^{21}, P P P C 1 B^{22} S O S 2^{18}, M R A S^{23}, R R A S^{24}$ and $L Z T R 1^{18,25}$ although not all of them have the same gene-disease association supporting evidences ${ }^{26}$.

At an early age, uncertain clinical diagnostics can emerge due to the presence of clinical manifestations that are common to NF1 as well as to other RASopathies or to other CALM-associated diseases. One example is the solely and combined presence of CALMs and skin fold freckling in LS and NF1 affected children ${ }^{27-29}$. Thus, children sharing a similar initial clinical presentation can bear mutations in different genes that predispose to very different clinical courses, that need to be managed in distinct ways. In this context, genetic testing can help in confirming a 
clinical suspicion, facilitating an early adequate surveillance. For instance, genetic testing has been recommended to confirm NF1 in children fulfilling only pigmentary features of the diagnostic criteria ${ }^{30}$.

Current comprehensive genetic testing strategies for these inherited diseases consist in either multi-gene panels or whole exome sequencing to solve problems like genetic heterogeneity or inconclusive clinical presentations ${ }^{31}$. In addition, due to the power and cost-effectiveness of NGS-based strategies and the demonstrated clinical utility ${ }^{32}$, inconclusive but suggestive clinical presentations are increasingly being accepted for genetic testing, accounting for a significant raise in genetic tests, especially at pediatric ages ${ }^{33}$. On the other side of the NGS coin, there is the exponentially growing number of identified genetic variants and the problem related to their pathogenicity analysis and interpretation. To standardize this process, the ACMG/ AMP guidelines ${ }^{34}$ are now widely adopted into clinical practice. However, these guidelines need to be adjusted according to the specificities of the genetic conditions being tested. In the case of RASopathies, an additional complication is the existence of diseases caused by both loss-offunction and gain-of-function mutations. Recently, the ClinGen RASopathy Expert Panel has published new guidelines to improve gene-disease association and variant interpretation for Noonan, NSML, Costello, CFC and Noonan-like syndromes ${ }^{26,35}$.

In this study, a new version of the I2HCP (ICO-IMPPC Hereditary Cancer Panel), a custom NGS-based diagnostic strategy ${ }^{36}$, was validated for its use in the molecular testing of all RASopathy-related genes. Once validated, the performance and implementation of this panel into routine diagnostics was evaluated for its use in the genetic testing of two groups of individuals sent to the diagnostics lab with an inconclusive clinical diagnostic: patients with a clinical suspicion of a RASopathy or children presenting multiple CALMs. We describe here a mutational spectrum for the two groups of individuals and demonstrate the appropriateness of our NGS panel in this clinical scenario. 


\section{MATERIALS \&METHODS}

\section{$\underline{\text { Subjects }}$}

All procedures performed were in accordance with the ethical standards of the IGTP Institutional Review Board, who approved this study, and with the 1964 Helsinki declaration and its later amendments. Informed consent was obtained from all genetically diagnosed individuals that were included in the study. Genomic DNA from 259 unrelated individuals were obtained from blood lymphocytes using standard protocols at three different centers: Department of Human Genetics KU Leuven; Department of Genetics, Microbiology and Statistics University of Barcelona and Genetics Testing Unit of IGTP. The 259 samples were grouped in four different sample sets: 1) RASopathy control group; 2) NF1 control group; 3) RASopathy-like group and 4) multiple CALM phenotype group. The RASopathy control set comprised 29 samples with variants in genes responsible for: Noonan syndrome, NSML, Costello, CFCS, NF1 and Legius syndrome, previously tested in KU Leuven and University of Barcelona. All samples had been genetically tested using pre-NGS workflows (cDNA and DNA Sanger sequencing). The RASopathy-like set consisted in 48 individuals with a clinical suspicion of a RASopathy-related syndrome (normally exhibiting facial dysmorphism and/or incomplete RASopathy-like traits), 16 of them previously tested in the University of Barcelona with no pathogenic mutation detected and 32 that arrived to the Genetics Testing Unit of IGTP. The NF1 control set contained 80 individuals fulfilling NF1 NHI criteria and the multiple CALM phenotype set comprised 102 children that presented more than 5 CALMs sent for testing at IGTP.

\section{Sample preparation and sequencing}

Library preparation and enrichment was performed as previously described ${ }^{36}$. Briefly, sample preparation was performed following the SureSelect XT protocol for MiSeq and enriched with custom I2HCP v2.2 library baits, which contained 135 genes including 22 genes involved in the RAS/MAPK pathway (Supplementary Table S1). 16 equimolar indexed samples were pooled after capture and sequenced in a MiSeq (Illumina) with Reagent Kit v3, $2 \times 300$. For each gene, we defined the regions of interest (ROIs) ${ }^{36}$. NGS data were processed and filtered according to the clinical indication using a custom data analysis pipeline as previously described ${ }^{36}$. The validation analysis of I2HCP was performed blindly using the RASopathy control group.

\section{Validation by Sanger Sequencing}

Any ROI for all genes tested with at least one base below 30x was Sanger sequenced using standard protocols (primer sequences available upon request). All pathogenic variants and VUS, were also validated by Sanger sequencing. Human Genome Variation Society (www.hgvs.org) nomenclature guidelines were used to name the mutation at the DNA level and the predicted resulting protein.

\section{NF1 and SPRED1 additional mutational analysis}

In NF1 control patients and in individuals with multiple CALM phenotype (or NF1-associated tumors) that tested negative with the custom I2HCP panel, NF1 and SPRED1 genes were additionally tested by MLPA (SALSA MLPA 
P122, P081, P082 and P295, following manufacturer's instructions). In addition, negative MLPA cases and those with inconclusive NF1 variants were further analyzed at RNA level. The entire coding region of NF1 was amplified from cDNA in five overlapping PCR fragments. PCR products were analyzed by electrophoresis and Sanger sequencing. A schematic representation of the whole testing workflow is represented in Figure 1.

\section{Variant analysis and interpretation}

We evaluated SIFT, PolyPhen, CADD, PROVEAN, MutationTaster, REST, VEST3 and MetaSVM. Some of them had a pre-defined score, while for CADD, REST and VEST3 we used scores higher than 19, 0.5 and 0.8 , respectively, to identify variants likely pathogenic. To evaluate in silico predictors we analyzed 45 recurrent NF1 missense variants $(\geq 3 \mathrm{x})$ reported at https://databases.lovd.nl/shared/variants/NF1/unique and 502 unique missenses in NS, NSML, Costello, CFC -causing genes. RASopathy missense variants were selected if reported three or more times in NSEuronet database (http://nseuronet.com) as VUS, pathogenic or likely pathogenic variants; or reported as pathogenic or likely pathogenic in ClinVar. All missense variants detected in this study were classified using the combination of SIFT, PolyPhen, CADD, PROVEAN and MutationTaster. In addition, FoldX ${ }^{37}$ was used to evaluate the structural impact of each variant detected in this study on the corresponding protein following developer's instructions (http://foldxsuite.crg.eu). Values higher than 0.8 were considered to destabilize significantly the entropic energy of analyzed proteins. InterVar (http://wintervar.wglab.org/) was also used to classify all VUS identified following published recommendations ${ }^{38}$.

\section{$\underline{\text { Statistical analysis }}$}

We tested whether the frequency of NF1 missense variants identified in the NF1 control and in the multiple CALM phenotype groups were different by applying a Chi square test using the Rscriptchisq.test function. 


\section{RESULTS}

\section{Validation of the I2HCP NGS-panel for the genetic analysis of RASopathy genes}

The I2HCP strategy was first developed and validated for testing hereditary cancer genes, showing an analytical sensitivity of $98.4 \%$ and an analytical specificity of $100 \%^{36}$. Our I2HCP NGS panel also contained 22 known RASopathy associated genes. We first evaluated the performance of the I2HCP for the genetic testing of RASopathy syndromes. We analyzed a control group of 29 samples (Table 1) with 28 known mutations in 15 RASopathy genes: 21 independent disease-causing mutations, 7 variants of unknown significance (VUS) and one case without a previously identified mutation. Although I2HCP custom panel v2.2 contained 135 genes, the genetic diagnostics strategy consisted in focusing the variant analysis only on those genes that have been consistently associated with the different RASopathy conditions (Supplementary Table S1). These genes included those recommended by the ClinGene RASopathy Expert Panel ${ }^{26}$, with the addition of NF1, SPRED1 and $C B L^{11}$, and RASA1 for its association with CM-AVM ${ }^{11}$. The whole validation process was performed blindly.

Each sample produced $3.1 \times 10^{6} \pm 0.67 \times 10^{6}$ SD paired reads, the mean depth of coverage was $530 \pm 116 \mathrm{SD}$, the coverage uniformity was of $35.2 \% \pm 1.9 \% \mathrm{SD}$, and $98.9 \% \pm 0.2 \%$ SD of the targeted bases were covered $\geq 30 \mathrm{x}$ (Supplementary Table S2, Supplementary Figure S1), very similar to the performance achieved for hereditary cancer genes $^{36}$. The I2HCP panel identified all previously known variants present in the validation set, including substitutions and small insertions/deletions. No VUS or pathogenic variant was identified in the case in which no mutations were previously identified (Table 1). Therefore, considering the 28 known variants present in the validation set, there was an excellent performance of the I2HCP for testing RASopathy genes, preserving the same sensibility and specificity as reported previously in the validation of I2HCP panel for hereditary cancer testing ${ }^{36}$.

\section{Implementation of I2HCP into routine genetic testing for RASopathies and children with multiple CALMs}

We used the I2HCP-RASopathy subpanel to genetically test two groups of patients that at early childhood can have an overlapping presentation of clinical manifestations with NF1. These were basically grouped by phenotype: 1) individuals with a clinical suspicion of a RASopathy; 2) children with multiple CALMs. After clinical evaluation of individuals, sample preparation, sequencing and data analysis were performed for the whole set of 135 genes present in the panel. For the first group of patients, RASopathy genes $(n=15)$ were further analyzed up to the level of variant interpretation (Supplementary Table S1). Similarly, only genes that have been consistently related with a CALM phenotype were analyzed for children with multiple CALMs in a stepwise manner (Figure 1, Supplementary Table S1): first NF1 and SPRED1, if necessary, other RASopathy genes, and finally, after a careful evaluation and following Suerink M et al. (2019) recommendations ${ }^{9}$, other genes related with a multiple CALM-phenotype ${ }^{4,9}$.

\section{Interpretation of missense variants in RASopathy genes}


In order to classify missense variants in a consistent manner, we followed the recommendations of the ClinGen's RASopathy Expert Panel ${ }^{35}$. For genes not evaluated by this panel but included in this study we followed the ACMG/AMP guidelines ${ }^{34}$ (Supplementary Table S6). Among the criteria for the interpretation of variants, guidelines require the agreement of the in silico programs used. To establish the best in silico program combination for analyzing RASopathy genes, we first compiled two independent control data sets. They consisted in a list of 45 pathogenic or likely pathogenic missense variants for NF1 (LOVD NF1 database); and 502 variants from NSEuroNet database and ClinVar, for other RASopathy genes. We then evaluated the performance of three different in silico algorithm combinations with these two data sets. The first two included: SIFT, PolyPhen and CADD in one combination, and SIFT, PolyPhen, CADD, PROVEAN and MutationTaster in the other. Using both data sets, we found that more than $80 \%$ of pathogenic and likely pathogenic variants had a total concordance in both combinations, 38 out of 45 (84\%) for NF1 (Supplementary Table S2) and 482 out of 502 (96\%) for the other RASopathy genes (Supplementary Table S3). The results obtained using the 3-and 5-algorithm combinations were exactly the same and performed slightly better than previous findings using ClinVar data ${ }^{39}$. The third combination included three high-performance algorithm predictors: REVEL, VEST3 and MetaSVM. In this case, the rate of concordance between all three algorithms was of 36\% (16 out of 45) for NF1 dataset and 59\% (309 out of 521) for RASopathy dataset (Supplementary Table S2 and Table S3), performing worse than previously described ${ }^{39}$. Therefore, we chose the 5-algorithm combination for the interpretation of missense variants in NF1 and other RASopathy genes.

Using this algorithm, we still had a fair percentage of variants classified as VUS. To try improving their classification, we explored the possibility of using FoldX, a structure-energy-based predictor tested previously in the context of RASopathies ${ }^{37}$, and InterVar, a clinical semi-automatic interpreter of genetic variants based on ACMG/AMP guidelines for variant classification ${ }^{38}$. The information provided by FoldX was very interesting due to its complementarity, but the absence of 3D protein structures for a significant number of RASopathy gene products or domains limited its use. Furthermore, a fair proportion of pathogenic or likely pathogenic variants were classified as VUS by InterVar. In our hands, none of them added utility regarding variant interpretation in a routine diagnostics setting.

\section{Testing RASopathy-like patients}

A total of 48 patients composed the RASopathy-like set. Overall the I2HCP diagnostics strategy allowed to detect a disease-causing variant in about $44 \%$ of the cases ( $n=21 / 48$ ). In addition, in $12 \%$ of the individuals $(n=6)$ a VUS in one of the interrogated genes was identified. About $44 \%$ of the cases were negative after using the NGS RASopathy panel ( $\mathrm{n}=21)$ (Figure 2A, Supplementary Table S4). Sixteen out of 48 cases of the RASopathy set had previously been tested for a few genes causing Noonan and Costello syndromes, and represented patients that tested negative with previous approaches). Of those, we were able to detect a pathogenic variant in 5 cases (31\%, 5/16) and one VUS (6\%) in another individual. The remaining 32 were genetically analyzed for the first time. In this group, 16 disease-causing mutations were identified (50\%) and 5 VUS (16\%). 11 patients (34\%) tested negative for all 
RASopathy genes using the NGS-I2HCP panel strategy (Figure 2B). However, among the individuals that tested negative, we identified a deleterious PTEN mutation in two patients. In both cases, patients exhibited some clinical manifestations related to RASopathies but none of them fulfilled clinical criteria for any particular condition. All together, these results showed that I2HCP strategy exhibit a good diagnostics yield outperforming previous single gene-based strategies.

\section{Mutational spectrum by phenotype}

Some patients of the RASopathy set $(\mathrm{n}=18)$ arrived to the genetic diagnostics lab with a defined clinical suspicion of NS, NSML, CFCS or CM-AVM, supported by their clinical record, while others $(n=30)$ arrived with an inconclusive clinical diagnostic and where referred as RASopathy-like (Supplementary Table S4). We analyzed the mutational spectrum for each specific suspected condition, and for the RASopathy-like group (Figure 3, Supplementary Table S4). Among patients with a suspicion of a specific condition ( $\mathrm{n}=18), 5$ tested negative (28\%), 10 carried a pathogenic mutation (55\%) and 3 carried a VUS (17\%). Mutated genes matched their corresponding conditions, given the known genetic heterogeneity present in RASopathies. The only exceptions were two independent pathogenic variants identified in SPRED1 and NF1 in two patients of the NS group. The patient with the SPRED1 mutation was 22yo when tested and due to his/her facial traits was suspected to have NS. The patient with the NF1 mutation was a child first diagnosed at the age of 10 months with a clinical presentation that resembled a RASopathy without CALMs.

The mutational spectrum in the RASopathy-like group (Figure 3, inconclusive phenotype) was similar to the one considering the whole RASopathy set (Figure 2A) being NF1, PTPN11 and RAF1 the genes concentrating most of the disease-causing mutations identified. Among the patients that tested negative in this group, there were the two individuals with a pathogenic mutation in PTEN.

If disease-causing mutations and VUS were considered together, NF1 was the gene with more genetic variants identified (n=7) in the RASopathy-like group, even higher than PTPN11 (n=5) (Figure 2B), although any bias in the selection of individuals arriving to our testing unit could greatly influence this picture. Within those patients with a clinical suspicion of a RASopathy and an NF1 mutation, we realized that two carried a pathogenic variant in NF1 but also bore a missense variant classified as VUS at the NF1 GRD domain (patients 469 and 711) (Supplementary Table S4). Both were children exhibiting Noonan-like facial dimorphism, ptosis, macrocephaly, developmental delay, and learning problems among other RASopathy features. Another patient of the RASopathy-like group (patient 464) carried two missense variants at RIT1 in trans, one pathogenic and the other one classified as VUS, and showed a severe RASopathy-like phenotype (Supplementary Table S4). We were not able to perform segregation analysis for any of the VUS identified in these three patients.

\section{Testing children with multiple CALMs}

The I2HCP NGS panel allowed the genetic testing of multiple-CALM phenotype in a stepwise manner as mentioned above (Figure 1). We analyzed NF1 and SPRED1 genes in 102 children that arrived from different centers to our 
genetic testing unit with clinical suspicion of NF1 or LS due to the presence of multiple CALMs. None of them had been previously genetically analyzed and represented sporadic cases. We further divided the 102 children in three subsets: children exhibiting $\geq 6$ CALMs as the only clinical manifestation ( $\mathrm{n}=71$ ); children with multiple CALMs and another clinical manifestations not being an NF1 NIH clinical criteria (facial dimorphism, cognitive disorders or tumors not related to NF1) (n=26), and finally, a small group of 5 infants with multiple CALMs and skin-fold freckling. In addition, we also analyzed 80 patients fulfilling NF1 NIH criteria by the presence of multiples CALMs (with or without skin-fold freckling) and at least one additional NF1 NIH criteria that we used as a control group for an NF1 phenotype. In all 182 cases we applied the I2HCP NGS-based strategy for NF1 and SPRED1 analysis followed by MLPA of both genes and RNA-based (cDNA) analysis of NF1, when required.

As a baseline for comparison, we first analyzed the performance of the new testing workflow in the group fulfilling NF1 NIH clinical criteria ( $\mathrm{n}=80$ ) (Figure 1, Supplementary Figure S2). The use of the I2HCP panel alone detected a potentially pathogenic NF1 variant in 71 individuals (71/80; 89\%). In 64 cases (64/80; 80\%) the variant was conclusively pathogenic and for seven cases bearing intronic or missense variants, additional RNA analysis was required to clarify their pathogenicity. Five of the seven variants altered the correct $N F 1$ splicing $(5 / 80,6.25 \%)$ and the two remaining ones were classified as VUS. Segregation analysis of these VUS did not clarify their pathogenicity. MLPA was further performed in nine cases in which no variant was identified by I2HCP NGS analysis. We identified two copy number variation (CNV) mutations (2/80; 2.5\%). Finally, RNA-based analysis of the seven remaining negative cases identified four independent deep intronic mutations affecting the correct NF1 splicing (4/80; 5\%). In three cases (3/80; 3.75\%) we were unable to identify a disease-causing mutation, although mosaicism cannot be discarded (Figure 4A, Supplementary Figure S2 and Table S5). In summary, I2HCP panel alone identified a pathogenic variant in 69/80 cases (86\%), although five cases required additional RNA-based analysis. MLPA analysis identified two further cases and RNA analysis was required to identify four additional deep-intronic mutations. Two variants were classified as VUS (2.5\%) and three cases tested negative (3.75\%). The use of the I2HCP panel helped in simplifying the whole testing process and had a good performance, similar to previous comprehensive strategies, although a combination of DNA and RNA-based analysis was still necessary to reach a high sensitivity.

We then analyzed the other cases with $\geq 6$ CALMs, grouped by phenotype. The percentage of positive tests varied among groups . Fifty-two percent (37/71) of children exhibiting only $\geq 6$ CALMs carried a disease-causing variant and in addition we detected a VUS in 12\% of them (9/71) (Figure 4A; Supplementary Table S5). In the group of children with $\geq 6$ CALMs and one sign not related to NF1, the percentage of positive tests was of $38 \%(10 / 26)$ and in $\sim 12 \%$ of cases a VUS was identified (3/26). A pathogenic mutation in the $N F 1$ gene was identified in all cases $(n=5)$ exhibiting $\geq 6$ CALMs and skin-fold freckling as the only clinical manifestations (Figure 4A). All nonconclusive cases (negative cases and cases with VUS for NF1 and SPRED1) from the different groups were analyzed for the rest of genes present in the RASopathy panel. In addition, in the specific individuals with $\geq 6$ CALMs and a non-NF1 tumor present, genes causing CMMRD were analyzed. No additional genetic variants were identified. 
NF1 mutational spectrum.

The new NF1 genetic testing workflow identified a genetic variant in 96\% of patients fulfilling NF1 NIH criteria by the presence of $\geq 6$ CALMs and two additional NIH criteria (in $92 \%$ of cases a conclusive pathogenic variant). In this group, we detected NF1 variants spread over the entire coding region of NF1, including nonsense (32.5\%), frameshift (27.3\%), missense variants (18.2\%, two of them VUS), NF1 microdeletions (1.3\%), intragenic CNV involving several exons (1.3\%), and variants in canonic and non-canonic splice sites and deep intronic mutations (19.4\%) (Figure 4B, Supplementary Table S5). 70 out of these 77 variants were unique in our set. The percentage of each mutation type was slightly different but in accordance to other published results ${ }^{40}$. Since we did not test the effect of all mutations at RNA level, the slight discrepancy regarding splicing mutations could come from the fact that different types of pathogenic mutations at DNA level can cause splicing defects ${ }^{41,42}$. Any bias in the NF1 population analyzed would also affect the frequency of the different mutation types. Remarkably, the different frequency of NF1 mutation types detected in the cohort of children with $\geq 6$ CALMs $(n=102)$ compared to the control NF1 group was statistically significant, consisting in a lower proportion of nonsense and frameshift mutations and a higher proportion of missense variants, both, clearly pathogenic and VUS (Figure 4B, Supplementary Table S5). 


\section{DISCUSSION}

In this study, we first validated the use of the I2HCP V2.2 diagnostic strategy, a custom NGS-based panel of 135 genes and an analysis pipeline already validated for hereditary cancer testing ${ }^{36}$, for the genetic diagnostics of patients with any RASopathy syndrome. The panel included 22 RASopathy-related genes. However, for its use in genetic testing, we considered only the 11 RASopathy genes recommended by the ClinGen RASopathy Expert Panel ${ }^{26}$ together with NF1, SPRED1, CBL and RASA1. The use of a smaller gene panel could be more cost-effective, however, the I2HCP was designed to fit our global diagnostics activity and provides a greater capacity of analyzing uncertain phenotypes potentially involving other genes included in this panel. The custom nature of I2HCP provides the flexibility for rapidly incorporating new recommended genes, like $P P P 1 C B$ and MRAS genes.

The sensitivity and specificity of I2HCP v2.2 to detect alterations in RASopathy genes using a control group of 29 RASopathy patients were similar to the ones reported for hereditary cancer genes ( $>99 \%)^{36}$. When applied to the RASopathy-like group (48 individuals), in $60 \%$ of the cases a clear pathogenic mutation (44\%) or a VUS (12\%) were identified (in $66 \%$ of the patients if only those tested for the first time were considered). The gene-specific frequencies detected were consistent with previous reports (NSEuronet) although the percentage of negative cases was slightly greater than reported ${ }^{43}$. However, the overall performance of the I2HCP testing strategy partially depended on the clinical evaluation of the patients referred for testing (see M\&M; Supplementary Table S4). As an example, in the RASopathy-like group we detected two patients with a pathogenic PTEN mutation. Nevertheless, this panel-based strategy outperformed previous testing single-gene strategies, since it was able to detect pathogenic variants in 5 individuals from a group of 16 patients that had been genetically tested previously.

If we just consider the testing for NF1 mutations, the use of the I2HCP panel helped in simplifying the whole testing algorithm and experimental procedures while preserving a good performance and diagnostic yield compared with pre-NGS diagnostic strategies. I2HCP mapping and variant calling constrictions avoided interference of NF1 pseudogenes ${ }^{36}$. However, we consider necessary the additional use of a CNV detection assay and an RNA-based analysis in the overall testing strategy. The use of the I2HCP panel alone identified a pathogenic variant in $86 \%$ of the NF1 control group (69/80 cases) although RNA-analysis was necessary for the correct assessment of functional impact in 5 cases. In addition, RNA-based analysis was indispensable to detect four additional cases bearing deepintronic mutations. Bioinformatic CNV analysis using panel-based NGS data has greatly improved. However, in our hands, the CNV analysis of the NF1 gene using panel data is still quite variable and is being validated for diagnostic purposes. Thus, all CNV analysis in the present work were performed by MLPA.

In the cohort of RASopathy-like patients, we detected seven patients with a NF1 mutation, thus with Neurofibromatosis Type 1. Most of them exhibited a Noonan-like facial dimorphism with or without other RASopathy clinical manifestations, and some of them presented CALMs like in Neurofibromatosis-Noonan syndrome (NF-NS, OMIM:\#601321). Pathogenic variants along the NF1 gene, including in-frame mutations in the GRD, have been associated with NFNS ${ }^{44}$. These findings highlight the difficulty of clinically discriminating among RASopathy conditions at pediatric ages and supports the recommendation of analyzing the classical RASopathy 
gene set together with NF1 and SPRED1 genes at the same time in order to increase the number of positive genetic tests in patients with a clinical suspicion of a RASopathy ${ }^{44,45}$.

The presence of $\geq 6$ CALMs of $0.5 \mathrm{~cm}$ could be indicative of NF1 but also of LS, CMMRD or other genetic conditions. In the analyzed cohort of 102 children with multiple CALMs, we only detected mutations in the NF1 and SPRED1 genes. Among the 102 children, the percentage of NF1 and LS patients were variable among the distinct subgroups established according to the presence or not of other clinical symptoms, but overall similar to previous reports ${ }^{28,46}$. In cases that tested negative for NF1 and SPRED1 mutations, we did not find alterations in other RASopathy genes in patients with $\geq 6$ CALMs and facial dimorphism or cognitive disorders, neither in MMR genes in the cases where this possibility was considered. Our results support previous recommendations for testing children with multiple CALMs (with or without other symptoms) in a stepwise manner ${ }^{9,30,47}$. In cases with CALMs and pediatric cancers or familial history of cancer, the genetic testing recommendations include the analysis of cancer predisposition genes $^{48}$. In these situations, the use of a panel such as the I2HCP could be convenient due to the possibility to analyze all recommended genes at once.

The NF1 mutational spectrum identified in the group of children with $\geq 6$ CALMs not fulfilling NF1 NIH criteria was different from the one present in the control NF1 group (Figure 4B). In the former group, the proportion of truncating mutations was lower than the control NF1 group. At the same time, the percentage of missense variants (both pathogenic and VUS) was higher, opening the possibility that these mutations could represent hypomorphic alleles generating incomplete or mild NF1 phenotypes. A prospective follow up of this group of patients could provide insight into this possibility and contribute to a better genotype-phenotype understanding.

Using the I2HCP strategy in hereditary cancer patients, we previously identified patients containing a complex variation landscape in herereditary cancer genes co-existing with the disease-causing mutation ${ }^{36}$. This was not the case for RASopathy-like individuals in RAS/MAPK pathway genes, except in three patients in which in addition to a pathogenic variant, a missense variant was detected in the same disease-causing gene (Supplementary Table S4 and Table S5). All three patients presented a complex clinical presentation, although the functional impact of these two co-existing variants in the same gene is unknown and further studies would be required to reach any conclusion. We followed the ACMG/AMP guidelines including modifications suggested by the ClinGen's RASopathy Expert Panel $^{35}$ for variant interpretation. ACMG/AMP guidelines recommend the agreement of all in silico programs tested for using them as supportive evidence. In our hands, SIFT, PolyPhen, CADD, PROVEAN and MutationTaster had the highest ratio of true concordance when they were combined, seeming the most suitable for the assessment of RASopathy-related variants. In any case, we found necessary to perform also a manual curation, in addition to in silico predicting algorithms, along the process of variant interpretation.

We also explored the possibility of using FoldX and InterVar for variant classification but, in our hands, none of them added utility regarding variant interpretation in a genetic testing context. For the whole set of 71 missense variants detected in both sets of patients only 25 of them (35\%) had an accurate 3D protein structure containing the variant we wanted to evaluate (Supplementary Table S6). In addition, $41 \%$ of pathogenic or likely pathogenic 
variants classified following our guidelines were classified as VUS by InterVar, exhibiting a poor correlation for its use.

We have validated the use of the custom I2HCP NGS panel for the routine genetic diagnostics of RASopathy-related genes. We have shown the utility of this strategy for testing children with an inconclusive clinical suspicion of a RASopathy and children with multiple CALMs. An NGS panel strategy outperforms previous testing strategies for these two phenotype-defined groups. We identified pathogenic variants in the NF1 gene in several inconclusive RASopathy cases. In addition, we identified pathogenic variants in NF1 and SPRED1 in more than 50\% of children with $\geq 6$ CALMs, exhibiting an NF1 mutational spectrum different from NF1 patients fulfilling NF1 NIH criteria.

\section{REFERENCES}

1. Huson SM, Compston DA, Clark P, Harper PS. A genetic study of von Recklinghausen neurofibromatosis in south east Wales. I. Prevalence, fitness, mutation rate, and effect of parental transmission on severity. $J$ Med Genet. 1989;26(11):704 LP - 711. doi:10.1136/jmg.26.11.704

2. DeBella K, Szudek J, Friedman JM. Use of the national institutes of health criteria for diagnosis of neurofibromatosis 1 in children. Pediatrics. 2000;105(3 Pt 1):608-614. doi:10.1542/peds.105.3.608

3. Ferner RE, Huson SM, Thomas N, et al. Guidelines for the diagnosis and management of individuals with neurofibromatosis 1. J Med Genet. 2007;44(2):81-88. doi:10.1136/JMG.2006.045906

4. Shah KN. The Diagnostic and Clinical Significance of Café-au-lait Macules. Pediatr Clin North Am. 2010;57(5):1131-1153. doi:10.1016/j.pcl.2010.07.002

5. Dos Santos ACE, Heck B, De Camargo B, Vargas FR. Prevalence of Cafi $\dot{c}^{1 / 2}$-au-Lait Spots in children with solid tumors. Genet Mol Biol. 2016;39(2):232-238. doi:10.1590/1678-4685-GMB-2015-0024

6. Evans DGR. What is the malignancy risk in neurofibromatosis type 1? J Clin Oncol. 2016;34(17):19671969. doi:10.1200/JCO.2016.66.8186

7. Ben-Shachar S, Dubov T, Toledano-Alhadef H, et al. Predicting neurofibromatosis type 1 risk among children with isolated café-au-lait macules. J Am Acad Dermatol. 2017. doi:10.1016/j.jaad.2017.02.027

8. Nunley KS, Gao F, Albers AC, Bayliss SJ, Gutmann DH. Predictive Value of Café au Lait Macules at Initial Consultation in the Diagnosis of Neurofibromatosis Type 1. Arch Dermatol. 2009;145(8):883-887. doi:10.1001/archdermatol.2009.169

9. Suerink M, Ripperger T, Messiaen L, et al. Constitutional mismatch repair deficiency as a differential diagnosis of neurofibromatosis type 1: Consensus guidelines for testing a child without malignancy. $J$ Med Genet. 2019;56(2):53-62. doi:10.1136/jmedgenet-2018-105664

10. Wimmer K, Rosenbaum T, Messiaen L. Connections between Constitutional Mismatch Repair Deficiency Syndrome and Neurofibromatosis Type 1. Vol 91.; 2017. doi:10.1111/cge.12904

11. Rauen KA. The RASopathies. Annu Rev Genomics Hum Genet. 2013;14(1):355-369. doi:10.1146/annurev-genom-091212-153523

12. Jindal G a., Goyal Y, Shvartsman SY, Burdine RD, Rauen K a. RASopathies: unraveling mechanisms with animal models. Dis Model Mech. 2015;8(9):1167-1167. doi:10.1242/dmm.022442

13. Upadhyaya M, Sarfarazi M, Huson SM, Stephens K, Broadhead W, Harper PS. Chromosome 17 markers and von Recklinghausen neurofibromatosis: A genetic linkage study in a British population. Genomics. 1987. doi:10.1016/0888-7543(87)90038-3

14. Brems H, Chmara M, Sahbatou M, et al. Germline loss-of-function mutations in SPRED1 cause a neurofibromatosis 1-like phenotype. Nat Genet. 2007. doi:10.1038/ng2113

15. Eerola I, Boon LM, Mulliken JB, et al. Capillary Malformation-Arteriovenous Malformation, a New Clinical and Genetic Disorder Caused by RASA1 Mutations. Am J Hum Genet. 2003. doi:10.1086/379793

16. Tartaglia M, Gelb BD, Zenker M. Noonan syndrome and clinically related disorders. Best Pract Res Clin Endocrinol Metab. 2011;25(1):161-179. doi:10.1016/J.BEEM.2010.09.002 
17. Aoki Y, Niihori T, Inoue SI, Matsubara Y. Recent advances in RASopathies. J Hum Genet. 2016;61(1):33-39. doi:10.1038/jhg.2015.114

18. Yamamoto GL, Aguena M, Gos M, et al. Rare variants in SOS2 and LZTR1 are associated with Noonan syndrome. J Med Genet. 2015:413-421. doi:10.1136/jmedgenet-2015-103018

19. Cordeddu V, Yin JC, Gunnarsson C, et al. Activating Mutations Affecting the Dbl Homology Domain of SOS2 Cause Noonan Syndrome. Hum Mutat. 2015. doi:10.1002/humu.22834

20. Chen P-C, Yin J, Yu H-W, et al. Next-generation sequencing identifies rare variants associated with Noonan syndrome. Proc Natl Acad Sci. 2014;111(31):11473-11478. doi:10.1073/pnas.1324128111

21. Vissers LELM, Bonetti M, Paardekooper Overman J, et al. Heterozygous germline mutations in A2ML1 are associated with a disorder clinically related to Noonan syndrome. Eur J Hum Genet. 2015;23(3):317324. doi:10.1038/ejhg.2014.115

22. Gripp KW, Aldinger K a., Bennett JT, et al. A novel rasopathy caused by recurrent de novo missense mutations in PPP1CB closely resembles Noonan syndrome with loose anagen hair. Am J Med Genet Part A. 2016;170(9):2237-2247. doi:10.1002/ajmg.a.37781

23. Motta M, Sagi-Dain L, Krumbach OHF, et al. Activating MRAS mutations cause Noonan syndrome associated with hypertrophic cardiomyopathy. Hum Mol Genet. May 2019. doi:10.1093/hmg/ddz108

24. Flex E, Jaiswal M, Pantaleoni F, et al. Activating mutations in RRAS underlie a phenotype within the RASopathy spectrum and contribute to leukaemogenesis. Hum Mol Genet. 2014;23(16):4315-4327. doi:10.1093/hmg/ddu148

25. Johnston JJ, van der Smagt JJ, Rosenfeld J a, et al. Autosomal recessive Noonan syndrome associated with biallelic LZTR1 variants. Genet Med. 2018;00(November 2017):1-11. doi:10.1038/gim.2017.249

26. Grant AR, Cushman BJ, Cavé H, et al. Assessing the gene-disease association of 19 genes with the RASopathies using the ClinGen gene curation framework. Hum Mutat. 2018;39(11):1485-1493. doi:10.1002/humu.23624

27. Stevenson D, Viskochil D. Pigmentary findings in neurofibromatosis type 1-like syndrome (Legius syndrome): Potential diagnostic dilemmas. JAMA - J Am Med Assoc. 2009;302(19):2150-2151. doi:10.1001/jama.2009.1690

28. Messiaen L, Yao S, Brems H, et al. Clinical and mutational spectrum of neurofibromatosis type 1-like syndrome. JAMA. 2009;302(19):2111-2118. doi:10.1001/jama.2009.1663

29. Stevens C a, Chiang P-W, Messiaen LM. Café-au-lait macules and intertriginous freckling in piebaldism: clinical overlap with neurofibromatosis type 1 and Legius syndrome. Am J Med Genet A. 2012;158A(5):1195-1199. doi:10.1002/ajmg.a.35297

30. Evans DGR, Salvador H, Chang VY, et al. Cancer and central nervous system tumor surveillance in pediatric neurofibromatosis 1. Clin Cancer Res. 2017;23(12):e46-e53. doi:10.1158/1078-0432.CCR-170589

31. Wright CF, FitzPatrick DR, Firth H V. Paediatric genomics: diagnosing rare disease in children. Nat Rev Genet. 2018;19(5):253-268. doi:10.1038/nrg.2017.116

32. Rehm HL, Bale SJ, Bayrak-Toydemir P, et al. ACMG clinical laboratory standards for next-generation sequencing. Genet Med. 2013;15(9):733-747. doi:10.1038/gim.2013.92

33. Adams DR, Eng CM. Next-Generation Sequencing to Diagnose Suspected Genetic Disorders. $N$ Engl $J$ Med. 2018;379(14):1353-1362. doi:10.1056/NEJMra1711801

34. Richards S, Aziz N, Bale S, et al. Standards and guidelines for the interpretation of sequence variants: a joint consensus recommendation of the American College of Medical Genetics and Genomics and the Association for Molecular Pathology. Genet Med. 2015;17(5). doi:10.1038/gim.2015.30

35. Gelb BD, Cavé H, Dillon MW, et al. ClinGen’s RASopathy Expert Panel consensus methods for variant interpretation. Genet Med. 2018;00(August 2017). doi:10.1038/gim.2018.3

36. Castellanos E, Gel B, Rosas I, et al. A comprehensive custom panel design for routine hereditary cancer testing: Preserving control, improving diagnostics and revealing a complex variation landscape. Sci Rep. 2017;7. doi:10.1038/srep39348

37. Kiel C, Serrano L. Structure-energy-based predictions and network modelling of RASopathy and cancer missense mutations. Mol Syst Biol. 2014;10(5):1-14. doi:10.1002/msb.20145092

38. Li Q, Wang K. InterVar: Clinical Interpretation of Genetic Variants by the 2015 ACMG-AMP Guidelines. Am J Hum Genet. 2017;100(2):267-280. doi:10.1016/j.ajhg.2017.01.004 
39. Ghosh R, Oak N, Plon SE. Evaluation of in silico algorithms for use with ACMG/AMP clinical variant interpretation guidelines. Genome Biol. 2017;18(1):1-12. doi:10.1186/s13059-017-1353-5

40. Messiaen LM and WK. NF1 mutational spectrum. Neurofibromatosis Kaufmann D (ed). 2008;16(i):63-77.

41. Ars E. Recurrent mutations in the NF1 gene are common among neurofibromatosis type 1 patients. $J$ Med Genet. 2003;40(6):82e - 82. doi:10.1136/jmg.40.6.e82

42. Sa A, Pros E, Ferna J, et al. Antisense Therapeutics for Neurofibromatosis Type 1 Caused by Deep Intronic Mutations Human Mutation. 2009. doi:10.1002/humu.20933

43. Tartaglia M, Gelb BD. Disorders of dysregulated signal traffic through the RAS-MAPK pathway: phenotypic spectrum and molecular mechanisms. Ann N Y Acad Sci. 2010;1214:99-121. doi:10.1111/j.1749-6632.2010.05790.x

44. De Luca A, Bottillo I, Sarkozy A, et al. NF1 gene mutations represent the major molecular event underlying neurofibromatosis-Noonan syndrome. Am J Hum Genet. 2005;77(6):1092-1101. doi:10.1086/498454

45. Ekvall S, Sjörs K, Jonzon A, Vihinen M, Annerén G, Bondeson M-L. Novel association of neurofibromatosis type 1-causing mutations in families with neurofibromatosis-noonan syndrome. Am J Med Genet A. December 2013. doi:10.1002/ajmg.a.36313

46. Bernier A, Larbrisseau A, Perreault S. Café-au-lait macules and neurofibromatosis type 1: A review of the literature. Pediatr Neurol. 2016;60:24-29e1. doi:10.1016/j.pediatrneurol.2016.03.003

47. Vasen HFA, Ghorbanoghli Z, Bourdeaut F, et al. Guidelines for surveillance of individuals with constitutional mismatch repair-deficiency proposed by the European Consortium "Care for CMMRD”(C4CMMR-D). J Med Genet. 2014;51(5):283-293. doi:10.1136/jmedgenet-2013-102238

48. Zhang J, Walsh MF, Wu G, et al. Germline Mutations in Predisposition Genes in Pediatric Cancer. $N$ Engl J Med. 2015;373(24):2336-2346. doi:10.1056/NEJMoa1508054 


\section{TABLES:}

Table 1: Validation of the I2HCP NGS panel for RASopathy testing.

Footnotes: depth: depth of coverage; freq: variant frequency; PAT: pathogenic; VLP: variant likely pathogenic; VUS: variant of unknown significance

\section{FIGURE LEGENDS}

Figure 1: Genetic testing workflow. Schematic representation of techniques used, genes analyzed and clinical manifestations considered.

Figure 2: Analysis of the Rasopathy-like group. The I2HCP diagnostics strategy allowed to detect a disease-causing variant in about $44 \%$ of the cases. A) Pie chart showing the percentage of positive cases, negative (light grey) and individuals with variants of unknown significance (VUS) (light blue). Positive cases are decomposed in the relative frequencies of pathogenic or likely-pathogenic variants for each RASopathy gene; B) Diagram showing the diagnostic yield and mutation frequencies for samples that had or had not been previously tested by pre-NGS methods. Dark blue indicates pathogenic or likely pathogenic variants detected, light blue VUS detected and light grey indicates negative cases.

Figure 3: Mutational spectrum of RASopathy-like individuals. Eighteen RASopathy-like patients (18/48) had a defined clinical suspicion of NS, NSML, CFCS or CM-AVM while 30 (30/48) had an inconclusive clinical diagnostic before genetic testing. For each group, the number of patients, the number of diagnostics with a pathogenic or likely pathogenic variant detected (+Test) and the number and frequency of mutations per gene is indicated.

Figure 4: Analysis of individuals with Multiple CALMs. Two groups of individuals were compared: an NF1 control set containing 80 individuals fulfilling NF1 NHI criteria, and a group of 102 children that presented more than 5 CALMs constituting the multiple CALM phenotype set. This latter set was further divided in three subsets depending on the presence (or not) of other clinical signs. A) Description of the mutational spectrum identified. Inserted pie charts indicate the percentage of individuals with identified variants per gene. B) Description of the NF1 mutational spectrum identified in each set and subset of individuals. C) Chi square test results evaluating the frequencies of NF1 missense variants in the NF1 control and in the multiple CALM phenotype groups. 


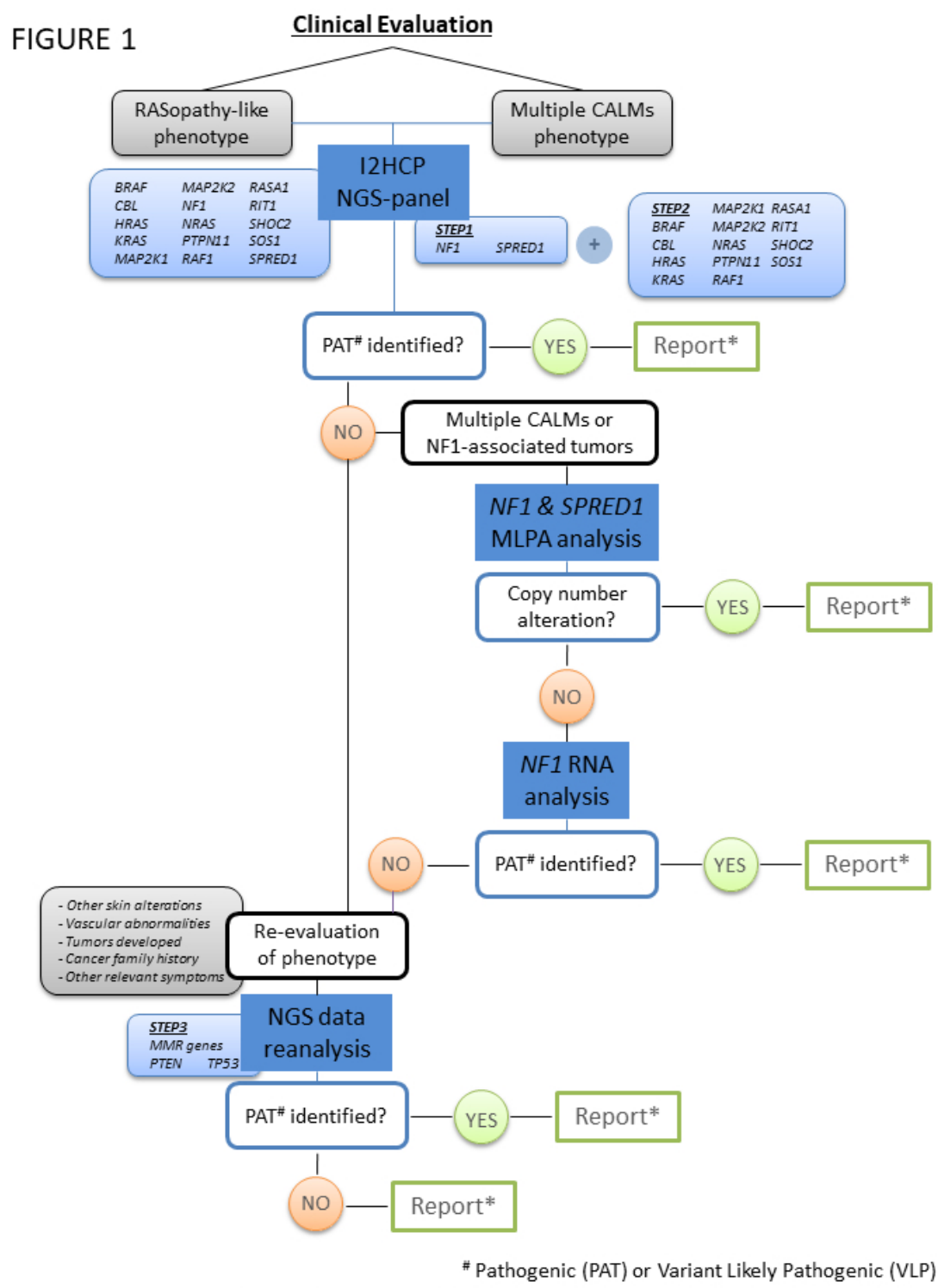

* PAT, VLP and VUS (Variant of Unknown Significance) validated by Sanger sequencing 
FIGURE 2

A.

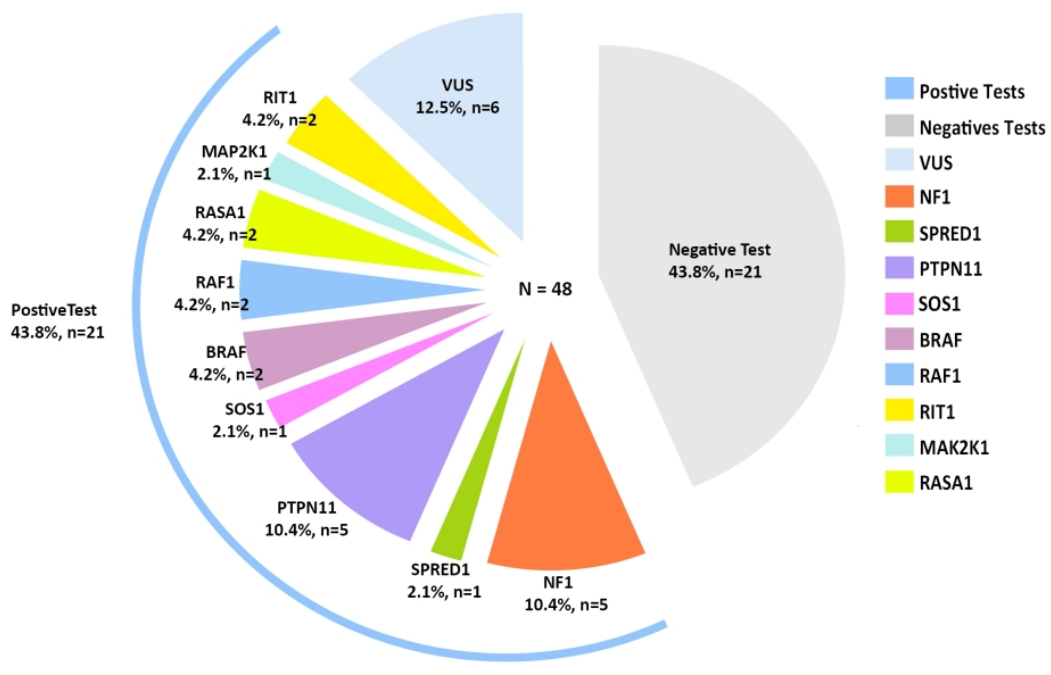

B.

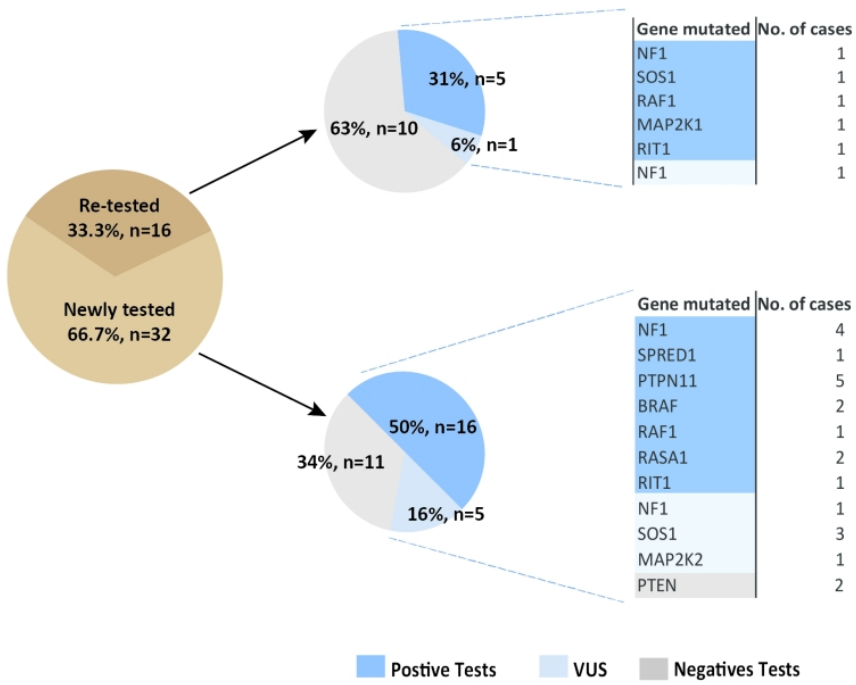

This article is protected by copyright. All rights reserved. 
FIGURE 3

\begin{tabular}{|c|c|c|c|c|c|c|}
\hline \multicolumn{2}{|c|}{ Phenotype } & No. patients & $\begin{array}{l}\text { No. } \\
+ \text { Test }\end{array}$ & \multicolumn{3}{|c|}{ Mutated genes } \\
\hline \multirow{4}{*}{ 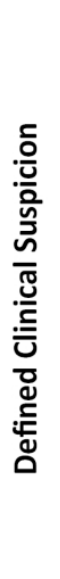 } & $\begin{array}{l}\text { Noonan- } \\
\text { like }\end{array}$ & $\begin{array}{l}n=10 \\
(21 \%)\end{array}$ & $\begin{array}{l}n=5 \\
(50 \%)\end{array}$ & $\begin{array}{lll}10 \% & \\
20 \% & 30 \% \\
\end{array}$ & \begin{tabular}{l|l}
3 Negatives & 2 PTPN11 \\
1 NF1 & 1 RIT1 \\
1 SPRED1 & 2 SOS1 Vus
\end{tabular} & \\
\hline & $\begin{array}{l}\text { CM-AVM } \\
\text { like }\end{array}$ & $\begin{array}{l}n=4 \\
(8 \%)\end{array}$ & $\begin{array}{c}n=2 \\
(50 \%)\end{array}$ & & \multicolumn{2}{|l|}{$\begin{array}{l}2 \text { Negatives } \\
2 \text { RASA1 }\end{array}$} \\
\hline & CFC - like & $\begin{array}{l}n=2 \\
(4 \%)\end{array}$ & $\begin{array}{c}n=2 \\
(100 \%)\end{array}$ & $100 \%$ & \multicolumn{2}{|l|}{2 BRAF } \\
\hline & $\begin{array}{l}\text { NSML- } \\
\text { like }\end{array}$ & $\begin{array}{l}n=2 \\
(4 \%)\end{array}$ & $\begin{array}{c}n=1 \\
(50 \%)\end{array}$ & $50 \%$ & \multicolumn{2}{|l|}{$\begin{array}{l}11 \text { PTPN11 } \\
1 \text { MAP2K2 VUS }\end{array}$} \\
\hline 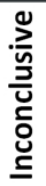 & RASo-like & $\begin{array}{l}n=30 \\
(62 \%)\end{array}$ & $\begin{array}{c}n=11 \\
(37.7 \%)\end{array}$ & $53.3 \%$ & 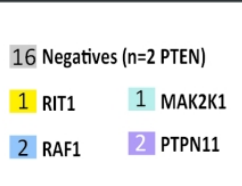 & $\begin{array}{l}3 \text { vUS (NF=2, SOS1=1) } \\
1 \text { SOS1 } \\
4 \text { NF1 }\end{array}$ \\
\hline
\end{tabular}


FIGURE 4

A.

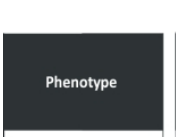

Only 26 CALMs

$\geq 6$ CALMs +1

26 CALMs +1
symptom (Tumor not NF1-related, reckling, cognitive disordes

26 CALMst skin-fold freckling

Control NF1 group Adults or children 26
CALMs +2 NIH criteria

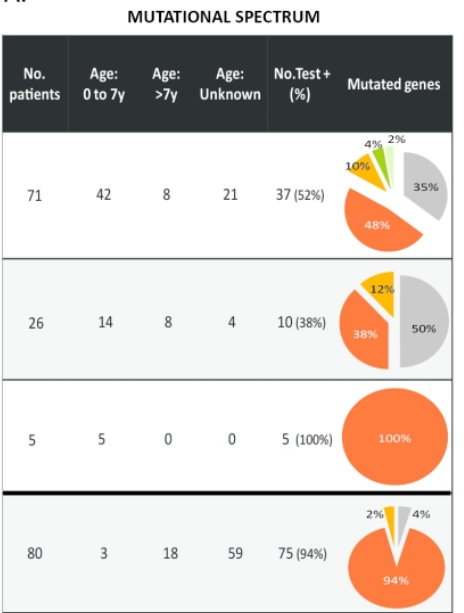

B.

B. NF1 MUTATIONAL SPECTRUM

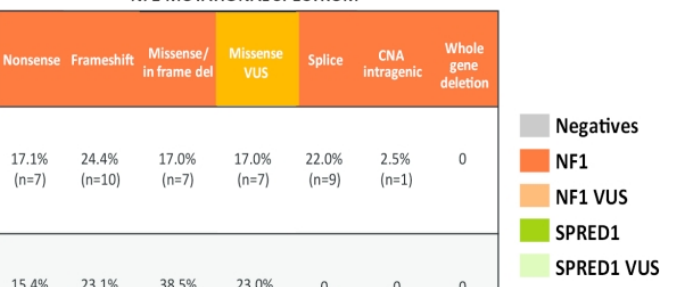

C.

Statistical analysis of missense frequencies

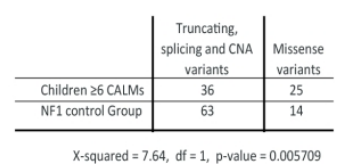




\section{(1) TABLE 1}

\begin{tabular}{|c|c|c|c|c|c|c|c|c|c|c|c|c|c|c|c|}
\hline Sample & Gene & NM & cDNA Annotation & $\begin{array}{l}\text { Exon } \\
\text { number }\end{array}$ & $\begin{array}{l}\begin{array}{l}\text { Protein } \\
\text { annotation }\end{array} \\
\end{array}$ & Genomic Position & depth & freq & dbSNP & Clinvar & $\begin{array}{l}\begin{array}{l}\text { Population } \\
\text { Freq Max* }\end{array} \\
\text { (EUR) }\end{array}$ & EXAC_nfe & PREDICTORS & $\begin{array}{l}\text { NSEuroNet } \\
\text { or LOVD }\end{array}$ & Classification \\
\hline 461 & HRAS & NM_005343.2 & $c .34 G>A$ & 2 & p.Gly12Ser & chrr11:534289 & 899 & 52,23 & rs104894229 & Costello: Pathogenic & & & PAT & $466 \mathrm{x}$ & VLP \\
\hline 466 & PTPN11 & NM_002834.3 & c. $1391 \mathrm{G}>\mathrm{C}$ & 12 & p.Gly464Ala & chr12:112926258 & 446 & 45,29 & rs121918469 & Noonan \& LEOPARD: Pathogenic & & & PAT & $8 \mathrm{x}$ & PAT \\
\hline 467 & BRAF & NM 004333.4 & c. $770 A>6$ & 6 & p.GIn257Arg & chr7:140501302 & 493 & 50,61 & rs 180177035 & CFC: Pathogenic & & & PAT & $67 x$ & PAT \\
\hline 473 & BRAF & NM_004333.4 & c. $1783 \mathrm{~T}>\mathrm{C}$ & 15 & p.Phe595Leu & chr7:140453152 & 353 & 46,18 & & & & & PAT & $7 x$ & PAT \\
\hline 475 & HRAS & NM 005343.2 & c. $35 \mathrm{G}>\mathrm{C}$ & 2 & p.Gly12Ala & chr11:534288 & 921 & 49,89 & rs104894230 & Costello: Pathogenic & & & & - & PAT \\
\hline 477 & BRAF & NM_004333.4 & c. $770 \mathrm{~A}>\mathrm{G}$ & 6 & p.Gln257Arg & chr7:140501302 & 388 & 48,71 & rs180177035 & Noonan \& CFC: Pathogenic & & & PAT & $67 x$ & PAT \\
\hline 483 & MAP2K1 & NM_002755.3 & c.389A $>G$ & 3 & p.Tyr130Cys & chr15:66729181 & 885 & 50,51 & rs121908595 & CFC: Pathogenic & & & PAT & $31 \times$ CFC & PAT \\
\hline 484 & BRAF & NM_004333.4 & c. $1574 \mathrm{~T}>\mathrm{A}$ & 13 & p.Leu525GIn & chr7:140476832 & 361 & 43,49 & & & & & PAT & $2 x$ & VUS \\
\hline 489 & BRAF & NM_004333.4 & c. $1801 \mathrm{~A}>\mathrm{C}$ & 15 & p.K601Gln & chr7:140453134 & 585 & 45,3 & & Noonan: Pathogenic & & & PAT & $3 \times$ CFC & VLP \\
\hline 525 & SPRED1 & NM_152594.2 & c.1149_1152delAGAG & 7 & p.Gly385Ilefs & chr15:38643679 & 519 & 47,59 & & & & & PAT & $15 x$ & PAT \\
\hline 543 & PTPN11 & NM_002834.3 & $c .836 \mathrm{~A}>\mathrm{G}$ & 7 & p.Tyr279Cys & chr12:112910827 & 162 & 52,47 & rs121918456 & Noonan \& LEOPARD: Pathogenic & & & & $75 x$ & PAT \\
\hline 544 & PTPN11 & NM_002834.3 & c. $922 \mathrm{~A}>\mathrm{G}$ & 8 & p.Glu308Asp & chr12:112915523 & 390 & 45,38 & rs28933386 & Noonan \& LEOPARD: Pathogenic & 0,0001 & 0,0000184 & INCONGRUOUS & $195 x$ & PAT \\
\hline 545 & sos1 & NM 005633.3 & c. $2536 \mathrm{G}>\mathrm{A}$ & 16 & p.Asp846Lys & chr2:39234309 & 314 & 46,82 & & Noonan: Pathogenic & & & INCONGRUOUS & $26 x$ & PAT \\
\hline 546 & sos1 & NM_005633.3 & c. $806 \mathrm{~T}>\mathrm{C}$ & 6 & p.Mey269Thr & chr2:39278343 & 441 & 48,75 & & Noonan: Pathogenic & & & PAT & $20 x$ & PAT \\
\hline 547 & KRAS & NM 033360.2 & c. $179 \mathrm{G}>\mathrm{T}$ & 3 & p.Gly60Val & chr12:25380279 & 188 & 46,28 & & & & & PAT & - & VLP \\
\hline 548 & RAF1 & NM_002880.3 & c. $1472 C>T$ & 14 & p.Thr491lle & chr3:12627244 & 457 & 46,61 & rs80338799 & LEOPARD: Pathogenic & & & PAT & $2 x$ & VLP \\
\hline 549 & NF1 & NM_000267.3 & c.3508 3509insA & 27 & p.His1170fs & chr17:29560031 & 403 & 49,38 & & & & & & $\therefore$ & PAT \\
\hline 550 & RIT1 & NM_006912 & $c .284 G>C$ & 5 & p.Gly95Ala & chr1:155874247 & 658 & 46,96 & & Noonan: Pathogenic & & & PAT & $10 x$ & PAT \\
\hline 551 & \multicolumn{15}{|c|}{ No previous variant detected } \\
\hline 552 & sos2 & NM_006939.2 & c. $1127 \mathrm{C}>\mathrm{G}$ & 9 & p.Thr376Ser & chr14:50628269 & 804 & 51,37 & & & & & INCONGRUOUS & $9 x$ & VLP \\
\hline 553 & LZTR1 & NM_006767.3 & c. $2090 \mathrm{G}>\mathrm{A}$ & 18 & p.Arg697GIn & chr22:21350272 & 728 & 44,84 & & & & 0,00003681 & PAT & - & vUS \\
\hline 554 & NRAS & NM_002524.4 & $c .101 C>T$ & 2 & p.Pro34Leu & chr1:115258681 & 505 & 47,52 & & Epidermal_nevus: Pathogenic & & & PAT & $5 x$ as VUS & VUS \\
\hline 555 & NRAS & NM_002524.4 & c.101C $>T$ & 2 & p.Pro34Leu & chr1:115258681 & 495 & 44,85 & & Epidermal_nevus: Pathogenic & & & PAT & $5 x$ as VUS & VUS \\
\hline 556 & RAF1 & NM_002880.3 & c. $781 \mathrm{C}>\mathrm{T}$ & 7 & p.Pro261Ser & chr3:12645688 & 321 & 41,74 & rs 121434594 & Noonan: Pathogenic & & & PAT & $15 x$ & VLP \\
\hline 557 & LZTR1 & NM_006767.3 & c. $356 \mathrm{~A}>\mathrm{G}$ & 4 & p.Tyr119Cys & chr22:21341828 & 331 & 41,95 & & & & & PAT & $1 \mathrm{x}$ & VUS \\
\hline 558 & A2ML1 & NM_144670.4 & $\begin{array}{l}\text { C. } 1492 \mathrm{~A}>\mathrm{T} \\
\end{array}$ & 13 & p.Ser498cys & chr12:8998053 & 360 & 45,28 & & & & & BENIGN & - & vUS \\
\hline 559 & RRAS & NM_006270.3 & c. $491 \mathrm{~A}>\mathrm{C}$ & 5 & p.His164Pro & chr19:50139072 & 155 & 9,03 & & & & & BENIGN & $\therefore$ & vUS \\
\hline 560 & KRAS & NM_004985.3 & c. $458 \mathrm{~A}>\mathrm{T}$ & 5 & p.Asp153Val & chr12:25362838 & 300 & 47,67 & rs104894360 & CFC: Pathogenic & & & INCONGRUOUS & $13 x$ & PAT \\
\hline 561 & MAP2K1 & NM_002755.3 & c. $199 \mathrm{G}>\mathrm{A}$ & 2 & p.Asp67Asn & chr15:66727483 & 530 & 51,61 & & Noonan: Pathogenic & & & PAT & $4 \times$ CFC & VLP \\
\hline
\end{tabular}

* Maximum population frequency detected in 1000G, EXAC, Esp6500, CG46 